\title{
Prevalência e fatores associados a problema crônico de coluna em mulheres em idade fértil
}

\author{
Prevalence and factors associated with chronic back problem \\ in women of childbearing age
}

Camila Vasconcelos de Arruda Oliveira (https://orcid.org/0000-0002-5964-819X) ${ }^{1}$

Damião Ernane de Souza (https://orcid.org/0000-0002-2038-9397) ${ }^{2}$

Adriana Gomes Magalhães (https://orcid.org/0000-0002-0279-5930) ${ }^{1}$

Janaína Paula Costa da Silva (https://orcid.org/0000-0003-3801-6967) ${ }^{3}$

Grasiéla Nascimento Correia (https://orcid.org/0000-0002-2722-5205) ${ }^{1}$

${ }^{1}$ Faculdade de Ciências da Saúde do Trairi, Universidade Federal do Rio Grande do Norte. R. Trairi s/n, Centro. 59200000 Santa Cruz RN Brasil. camila.v.arruda@gmail.com ${ }^{2}$ Instituto Brasileiro de Geografia e Estatística

${ }^{3}$ Faculdade de Medicina, Universidade Federal de Uberlândia.

\begin{abstract}
Chronic Back Problem (CBP) is a public health concern. In Brazil, data from the $\mathrm{Na}$ tional Health Survey (PNS) estimated 27.0 million people (18.5\%) who reported CBP, affecting more women than men. This study aims to identify the factors associated with CBP among women of childbearing age. It is a cross-sectional study carried out with data from the PNS, where the dependent variable was the prevalence of $C B P$, and associated factors included socio-demographic items, life habits, reproductive history, nutritional status, diagnosis of depression and health perception. A total of 22,621 women aged 18 to 49 years were interviewed. Of these, $14.8 \%$ reported having CBP. The risk factors studied were: increased age; living with spouse; multiparity; smoking; overweight or obese, having Waist Circumference (WC) above $80 \mathrm{~cm}$ and Circumference/Height $(C / E)$ index above 0.5; negative self-perception of health; and depression. The only protective factor associated with CBP risk reduction was education level. We conclude that age, living with a spouse/ partner, smoking, multiparity, being overweight or obese, increased risk for cardiovascular diseases, depression and negative self-perceived health are associated with the development of $C B P$ in women of childbearing age.
\end{abstract}

Key words Chronic back pain, Spine, Women
Resumo No Brasil, dados da Pesquisa Nacional de Saúde (PNS) estimaram 27,0 milhões de pessoas $(18,5 \%)$ que referiram Problema Crônico de Coluna (PCC), afetando mais mulheres que homens. Este trabalho tem o objetivo de identificar, entre as mulheres em idade fértil, os fatores associados ao PCC. Trata-se de um estudo transversal realizado com dados da PNS, em que a variável dependente foi a prevalência de PCC, enquanto que os fatores associados incluiram itens sociodemográficos, hábitos de vida, histórico reprodutivo, estado nutricional, diagnóstico de depressão e percepção de saúde. Foram avaliadas 22.621 mulheres com idade entre 18 e 49 anos $e$, destas, $14,8 \%$ referiram ter PCC. Os fatores de risco estudados foram: aumento da faixa etária; viver com cônjuge/companheiro; multiparidade; ser tabagista; sobrepeso ou obesidade, ter Circunferência da Cintura (CC) acima de $80 \mathrm{~cm}$ e indice Circunferência/Estatura (C/E) acima de 0,5; autopercepção de saúde negativa; e diagnóstico de depressão. O único fator de proteção encontrado foi escolaridade. As associações observadas concluem que idade, viver com cônjuge/companheiro, tabagismo, multiparidade, sobrepeso e obesidade, risco aumentado para doenças cardiovasculares, diagnóstico de depressão e autopercepção de saúde negativa estão associados ao desenvolvimento de PCC em mulheres de idade fértil.

Palavras-chave Dor crônica, Coluna vertebral, Mulheres 


\section{Introdução}

O problema crônico de coluna (PCC) apesar de não ser fatal, se constitui um importante problema de saúde pública' ${ }^{1-3}$, econômico e social ${ }^{4,5}$. Também denominados de 'dores nas costas', o problema crônico de coluna engloba as cervicalgias, dores torácicas, ciáticas, transtornos dos discos intervertebrais, espondiloses, radiculopatias, e as dores lombares ${ }^{6}$.

No Brasil, segundo dados da Pesquisa Nacional de Saúde (PNS, 2013) ${ }^{7}$, foram estimados aproximadamente 27,0 milhões de pessoas de 18 anos ou mais de idade (18,5\%) que referiram PCC, dado similar ao observado em outros países ${ }^{8}$, sendo mais prevalente entre as mulheres e entre indivíduos com idade acima de 60 anos de idade. De acordo com Romero et al. ${ }^{9}$, a idade média de início das queixas de PCC no Brasil é aos 35 anos e chega a acometer até $28,1 \%$ da população com 60 anos?.

Considerada uma das queixas comumente relatadas pela população adulta, o PCC pode gerar incapacidade, redução da funcionalidade, e afastamentos do trabalho ${ }^{10}$. Por conseguinte, compromete a qualidade da vida, acarreta maior busca por atendimento médico ${ }^{3,11}$, e tem sido uma das principais causas de aposentadoria por invalidez ${ }^{12}$.

A literatura indica um conjunto de fatores associados aos PCC, como os sociodemográficos que incluem idade, sexo, renda e escolaridade; comportamentais que se refere ao tabagismo, sedentarismo, exposições ocorridas nas atividades cotidianas, como trabalho físico extenuante, vibração, posição viciosa, movimentos repetitivos; além de outros fatores, por exemplo a obesida$\mathrm{de}^{13}$.

Em conformidade em com os dados da PNS, diversos estudos internacionais ${ }^{14-17}$ apontam a desigualdade de gênero pelo PCC, tendo uma prevalência maior entre as mulheres, devido à constituição musculoesquelética e às atividades diárias desempenhadas por elas ${ }^{18}$. Osteoporose, menstruação, gravidez e fatores culturais também são fatores que podem estar relacionados com a maior prevalência de PCC entre as mulheres ${ }^{19}$.

É importante ressaltar que a vida da mulher é marcada por alterações anatômico-fisiológicas especificas, na puberdade, gestação e climatério ${ }^{20}$ que podem favorecer o surgimento de PCC. Além disso, a mulher durante a idade fértil geralmente tem dupla jornada de trabalho tendo que responder simultaneamente às demandas do trabalho remunerado e doméstico-familiar ${ }^{21}$, o que gera reflexos em sua saúde ${ }^{22}$, como a maior prevalência de Doenças Crônicas Não Transmissíveis (DCNT), dentre elas hipertensão arterial, PCC, depressão, artrite ou reumatismo e diabetes? ${ }^{7}$.

Embora o tema sobre PCC seja relevante, ainda não existem estudos representativos da população brasileira sobre a utilização de tratamentos para essa morbidade ${ }^{9}$. Segundo Frasson ${ }^{23}$, o tratamento conservador da dor lombar deve abordar, preferencialmente, tratamento medicamentoso, exercícios, terapia manual, educação, abordagem biopsicossocial e terapia cognitivo-comportamental.

Os tratamentos para o PCC geram ônus tanto para a saúde pública, uma vez que há necessidade de exames, medicamentos, fisioterapia, internações e cirurgias ${ }^{24,25}$, quanto para a economia, especificamente relacionado à Previdência Social, uma vez que há elevadas taxas de licenças de saúde devido afastamento do trabalho ${ }^{26} \mathrm{e}$ aposentadoria por invalidez relacionada à dor na coluna no Brasil ${ }^{12}$.

Dessa forma, o PCC representa um problema de saúde pública ${ }^{1-3}$ que tem impacto na vida profissional das pessoas que tem esse diagnóstico ${ }^{12}$, sobretudo em mulheres, devido aos fatores genéticos, físicos e culturais ${ }^{19}$ que às predispõem à morbidade. Assim, tendo em vista a relevância do tema em questão, a escassez de estudos no Brasil sobre o assunto, este trabalho tem o objetivo de identificar, entre as mulheres em idade fértil, os fatores associados ao PCC.

\section{Métodos}

Este é um estudo transversal realizado com dados da Pesquisa Nacional de Saúde (PNS) do módulo Estilos de Vida da PNS (2013) ${ }^{7}$. A PNS foi desenvolvida pelo Instituto Brasileiro de Geografia e Estatística (IBGE) em parceria com o Ministério da Saúde. A população de estudo foi composta por moradores de domicílios particulares do Brasil, exceto os localizados nos setores censitários especiais (quartéis, bases militares, alojamentos, acampamentos, embarcações, penitenciárias, colônias penais, presídios, cadeias, asilos, orfanatos, conventos e hospitais).

A amostragem da PNS é caracterizada por uma subamostra da amostra mestra do Sistema Integrado de Pesquisas Domiciliares - SIPD do IBGE, cuja abrangência geográfica é constituída pelos setores censitários da Base Operacional Geográfica do Censo Demográfico 2010, exceto 
aqueles com número muito pequeno de domicílios e os setores especiais.

A PNS teve amostra total de 60.202 pessoas maiores de 18 anos, o plano amostral empregado foi o de amostragem por conglomerado em três estágios de seleção (setores, famílias e indivíduos) ${ }^{27}$.

No primeiro estágio, a seleção das unidades primárias de análise foi obtida por amostragem aleatória simples selecionada previamente na amostra mestra. No segundo, foi selecionado por amostragem aleatória simples - um número fixo de domicílios particulares permanentes em cada uma das unidades primárias de análises selecionada no primeiro estágio. No terceiro estágio, dentro de cada domicílio da amostra, um morador com 18 anos ou mais de idade foi selecionado - também por amostragem aleatória simples - para responder à 3a parte (individual) do questionário. Essa seleção foi feita baseandose em uma lista de moradores elegíveis, construída no momento da entrevista ${ }^{27}$.

O questionário aplicado foi dividido em três partes, as duas primeiras destinadas a perguntas sobre características do domicílio, situação socioeconômica e de saúde dos moradores; a terceira foi individual e direcionada ao morador de 18 anos ou mais previamente selecionado, nela foram respondidas perguntas sobre morbidade $\mathrm{e}$ estilo de vida ${ }^{28}$.

Na PNS foram avaliadas mulheres de 18 a 101 anos. No presente estudo, foram consideradas mulheres em idade fértil na faixa etária de 18 a 49 anos, uma vez que a PNS não avaliou mulheres abaixo de 18 anos, limite superior à classificação de mulheres em idade fértil no Brasil, que é de 10 a 49 anos $^{29}$. Portanto, no total, foram avaliadas 22.621 mulheres em idade fértil de todo o Brasil.

A variável dependente analisada foi a prevalência de PCC, investigada por meio da pergunta: "O(a) sr.(a) tem problema crônico de coluna, como dor crônica nas costas ou no pescoço, lombalgia, dor ciática, problemas nas vértebras ou disco?", que tinha duas opções de resposta: sim ou não.

As variáveis independentes incluíram itens sociodemográficos, hábitos de vida, histórico reprodutivo, estado nutricional e diagnóstico de doenças, categorizadas da seguinte forma: a) Idade, em anos, 18-26, 27-32, 33-39, 40-49; b) Escolaridade: sem instrução, até o ensino fundamental completo, ensino médio incompleto e completo, ensino superior incompleto ou mais; c) Raça ou cor da pele: branca para as mulheres que se autodeclaram brancas, e preta, amarela, parda ou indígena outras para as mulheres que autodeclaram preta, amarela, parda ou indígena; d) Situação conjugal: viver ou não com cônjuge/ companheiro; e) Ocupação: ter ou não um trabalho.

Sobre os hábitos de vida como fatores de risco e proteção: a) Atividade física: ter praticado ou não atividade física nos último três meses e, além disso, foi construído um escore semanal de prática de atividade física, no qual o tempo despendido nas atividades foi multiplicado pelo número de dias e o ponto de corte foi a prática ou não de 150 minutos ou mais por semana ${ }^{30}$; b) Tratamento para PCC: ter realizado ou não fisioterapia por causa do PCC e ter tomado ou não injeção/medicação para o PCC; c) Tabagismo: hábito de fumar ou não; d) Assistir televisão: menos de 2 horas e maior ou igual a 2 horas.

Em relação ao histórico reprodutivo, a) Número de partos: nenhum, até dois, e três ou mais.

Quanto aos fatores de risco metabólicos, a) classificação da massa corporal utilizando o Îndice de Massa Corporal (IMC): magreza e eutrofia $\left(\leq 24.9 \mathrm{~kg} / \mathrm{m}^{2}\right)$, sobrepeso $(>24,99 \mathrm{e} \leq 29,99$ $\left.\mathrm{kg} / \mathrm{m}^{2}\right)$, obesidade grau I $(>29,99 \mathrm{e} \leq 34,99 \mathrm{~kg} /$ $\left.\mathrm{m}^{2}\right)$, obesidade grau II e III $\left(>34,99 \mathrm{~kg} / \mathrm{m}^{2}\right) \mathrm{b}$ ) circunferência da cintura $(\mathrm{CC})^{31}:$ c) Razão cintura/estatura $(\mathrm{C} / \mathrm{E})^{32}:<0,5$ risco diminuído para doenças cardiovasculares e $>0,5$ risco diminuído para doenças cardiovasculares.

Sobre o diagnóstico de doenças utilizou-se como parâmetro a depressão: a) diagnóstico ou não de depressão realizado pelo médico.

Na percepção do estado de saúde, utilizou-se autoavaliação de saúde estratificada da seguinte forma: muito boa e boa para as mulheres que autoavaliaram sua saúde como muito boa e boa, e regular, ruim e muito ruim para as mulheres que autoavaliaram sua saúde como regular, ruim e muito ruim.

A PNS foi aprovada pela Comissão Nacional de Ética em Pesquisa (Conep) do Conselho Nacional de Saúde (CNS). Todos os indivíduos entrevistados foram consultados, esclarecidos e aceitaram participar da pesquisa mediante assinatura de um Termo de Consentimento Livre e Esclarecido.

Realizou-se uma análise descritiva na qual foram estimadas frequências simples e relativas das variáveis independentes e dependente de acordo com as covariáveis do estudo. As variáveis foram descritas por meio de proporções. Foram estimadas a prevalência e razões de prevalência de apresentar problema crônico de coluna de acordo com as covariáveis por meio da regressão logística. 
A análise foi realizada no módulo survey para amostras complexas do Stata versão 9.0 (StataCorp., CollegeStation, EUA).

\section{Resultados}

Foram avaliadas 22.621 mulheres com idade entre 18 e 49 anos, entre as quais 3.355 (14,8\%) referiram ter PCC. A maioria das mulheres se autodeclararam como preta, amarela, parda ou indígena e outra $(62,7 \%)$, viviam com cônjuge ou companheiro $(61 \%)$, estudaram até o ensino médio completo $(40,5 \%)$ e trabalhavam no momento da avaliação (59,9\%) (Tabela 1).

Em relação aos hábitos de vida, maioria não era tabagista $(90,2 \%)$ e $80,6 \%$ eram sedentárias pois praticavam menos de 150 minutos de atividade física por semana (Tabela 1). Quanto ao estado nutricional, verificou-se $44,7 \%$ eram eutróficas e 31,3\% estavam com sobrepeso. A maioria das mulheres $(69,2 \%)$ possuíam elevado risco para doença cardiovascular de acordo a razão cintura/estatura ${ }^{32}$ (Tabela 1$)$.

Sobre as condições de saúde como histórico reprodutivo, verificou-se que a maioria das mulheres eram multíparas (50,3\%); no quesito da percepção de saúde, observou-se que a maioria das entrevistadas referiram ter saúde muito boa ou boa $(95,7 \%)$; e $91,7 \%$ das entrevistadas referiram não ter diagnóstico médico de depressão (Tabela 1).

Dentre as variáveis estudadas verificou-se que faixa etária (todas as faixas etárias acima de 27 anos, sendo mais elevada na faixa dos 40 aos 49 anos); viver com cônjuge/companheiro (OR $=1,13$; IC95\% $=1,04-1,22)$; ser tabagista $(\mathrm{OR}$ $=1,63$, IC95\% $=1,46-1,81)$; multiparidade $(\mathrm{OR}=\mathrm{IC} 95 \%=)$; ter sobrepeso $(\mathrm{OR}=\mathrm{IC} 95 \%$ $=$ ) ou obesidade $(\mathrm{OR}=\mathrm{IC} 95 \%=)$, ter CC acima de $80 \mathrm{~cm}$ e índice $\mathrm{C} / \mathrm{E}$ acima de $0,5(\mathrm{OR}=1,51$, IC95\% = 1,39 - 1,65); má percepção de saúde $(\mathrm{OR}=3,58$, IC95\% = 3,13-4,11); e diagnóstico de depressão $(\mathrm{OR}=\mathrm{IC} 95 \%=)$ são fatores de risco para o PCC em mulheres na idade fértil. O único fator de proteção ao PCC foi a escolaridade $(\mathrm{OR}=0,69 ;$ IC95\% $=0,60-0,80)$ (Tabela 2).

As variáveis raça/cor, trabalho, prática de atividade física nos últimos 3 meses, prática de atividade física semanal igual ou superior a 150 minutos, assistir TV por mais de duas horas não estiveram associadas com o desfecho (Tabela 2).

\section{Discussão}

Por meio dos dados da PNS verificou-se que das 22.621 mulheres em idade fértil avaliadas, 3.355 $(14,8 \%)$ referiram ter PCC. As características associadas à maior prevalência de algum PCC, com a regressão logística, foram: aumento da idade; viver com cônjuge ou companheiro, ser tabagista; multiparidade; ter sobrepeso e obesidade; ter CC acima de $80 \mathrm{~cm}$ e índice $\mathrm{C} / \mathrm{E}$ acima de 0,5 , ambos indicando maior risco de doença cardiovascular; saúde autorreferida como ruim, muito ruim ou regular quando comparada com avaliação muito boa e boa; e diagnóstico de depressão.

No presente trabalho, o aumento da faixa etária, sobretudo na faixa entre os 40 e os 49 anos $(\mathrm{OR}=2,70$, IC95\% $=2,42-3,03)$, indicou maior chance de desenvolver PCC, o que também foi observado em estudos com dados da Pesquisa Nacional por Amostra de Domicílios (PNAD) em 2003 e em $2008^{33,34}$. Esse fato pode ser devido às mudanças no organismo devido ao processo de envelhecimento, como redução da flexibilidade, problemas posturais, maior degeneração osteomuscular e, consequentemente, agravamento da dor ${ }^{35}$.

Outro fator risco para desenvolver o PCC, foi o relacionamento com cônjuge ou companheiro $(\mathrm{OR}=1,13$, IC95\% = 1,04 - 1,22). Não foram encontrados estudos que abordem diretamente a relação do PCC e relacionamento com cônjuge ou companheiro. Em estudo realizado por Dutra et al. ${ }^{36}$ é sugerido que essa relação pode se devida à dupla carga de trabalho, profissional e cuidado com a família e afazeres domésticos) que a mulher é submetida, principalmente quando tem um relacionamento estável.

Quanto ao tabagismo, verificou-se que as mulheres que fumam têm maior chance $(\mathrm{OR}=$ $1,63)$ de desenvolver PCC do que as que não fumam, resultado semelhante ao encontrado por Malta et al. ${ }^{35}(\mathrm{OR}=1,59$, IC95\% $=1,38-1,84)$. Atualmente o tabagismo é um reconhecido como fator de risco para doenças cardiovasculares ${ }^{37}$ e já foi também apontado como fator associado à percepção negativa da saúde ${ }^{37}$. Além disso, existem evidências de que fumantes e ex-fumantes têm maior predisposição a desenvolver dor crônica, uma vez que a nicotina ativaria o sistema imunológico, predispondo a dor lombar e as doenças reumáticas, entre outras condições ${ }^{38-40}$.

No que diz respeito ao histórico reprodutivo, observou-se que quanto maior o número de partos, a chance de apresentar PCC aumenta em $37 \%$. Essa associação também já foi encontrada 
Tabela 1. Características sociodemográficas, hábitos de vida, histórico reprodutivo, estado nutricional, diagnóstico de depressão e auto percepção de saúde de mulheres em idade fértil que referiram Problema Crônica de Coluna. Pesquisa Nacional de Saúde, Brasil, 2013.

\begin{tabular}{|c|c|c|}
\hline Variável & $\mathbf{N}$ & $\%$ \\
\hline \multicolumn{3}{|l|}{ Problema crônico de coluna } \\
\hline Não & 19.266 & 85,2 \\
\hline Sim & 3.355 & 14,8 \\
\hline \multicolumn{3}{|l|}{ Idade (anos) } \\
\hline $18-26$ & 5.033 & 22,3 \\
\hline $27-32$ & 5.648 & 25,0 \\
\hline $33-39$ & 5.614 & 24,8 \\
\hline 40 ou mais & 6.326 & 27,9 \\
\hline \multicolumn{3}{|l|}{ Raça/cor } \\
\hline Branca & 8.443 & 37,3 \\
\hline Preta, parda e outros & 14.178 & 62,7 \\
\hline \multicolumn{3}{|l|}{ Vive com cônjuge ou companheiro } \\
\hline Não & 8.830 & 39,0 \\
\hline Sim & 13.791 & 61,0 \\
\hline \multicolumn{3}{|l|}{ Escolaridade } \\
\hline Sem instrução & 2.027 & 9,0 \\
\hline $\begin{array}{l}\text { Até o ensino fundamental } \\
\text { completo }\end{array}$ & 8.096 & 35,8 \\
\hline Ensino médio completo & 9.173 & 40,5 \\
\hline $\begin{array}{l}\text { Ensino superior completo ou } \\
\text { mais }\end{array}$ & 3.325 & 14,7 \\
\hline \multicolumn{3}{|l|}{ Trabalho } \\
\hline Não & 9.074 & 40,1 \\
\hline Sim & 13.547 & 59,9 \\
\hline \multicolumn{3}{|c|}{ Atividade física nos últimos 3 meses } \\
\hline Não & 16.738 & 74,0 \\
\hline Sim & 5.883 & 26,0 \\
\hline \multicolumn{3}{|l|}{$\begin{array}{l}\text { Prática de } 150 \text { minutos de atividade } \\
\text { física por semana }\end{array}$} \\
\hline Não & 18.226 & 80,6 \\
\hline Sim & 4.395 & 19,4 \\
\hline \multicolumn{3}{|l|}{ Exercício ou fisioterapia para PCC } \\
\hline Não & 2.792 & 83,2 \\
\hline Sim & 563 & 16,8 \\
\hline \multicolumn{3}{|l|}{$\begin{array}{l}\text { Injeção ou outra medicação para } \\
\text { PCC }\end{array}$} \\
\hline Não & 2.022 & 60,3 \\
\hline Sim & 1.333 & 39,7 \\
\hline \multicolumn{3}{|l|}{ Tabagismo } \\
\hline Não & 20.392 & 90,2 \\
\hline $\operatorname{Sim}$ & 2.229 & 9,8 \\
\hline \multicolumn{3}{|l|}{ Assistir TV } \\
\hline$<2$ horas & 9.418 & 41,6 \\
\hline$\geq 2$ horas & 13.203 & 58,4 \\
\hline
\end{tabular}

Tabela 1. Características sociodemográficas, hábitos de vida, histórico reprodutivo, estado nutricional, diagnóstico de depressão e auto percepção de saúde de mulheres em idade fértil que referiram Problema Crônica de Coluna. Pesquisa Nacional de Saúde, Brasil, 2013.

\begin{tabular}{lrr}
\hline \multicolumn{1}{c}{ Variável } & N & \multicolumn{1}{c}{$\%$} \\
\hline Número de partos & & \\
$\quad 0$ & 636 & 2,8 \\
$\leq 2$ & 10.616 & 46,9 \\
$\quad \geq 3$ & 11.369 & 50,3 \\
IMC & & \\
$\quad \leq 24,99$ & 10.117 & 44,7 \\
$>24,99 \leq 29,99$ & 7.087 & 31,3 \\
$\quad>29,99 \leq 34,99$ & 3.086 & 13,6 \\
$\quad>34,99$ & 2.331 & 10,3 \\
Circunferência da cintura & & \\
$\quad<80$ & 7.026 & 31,1 \\
$\quad 80-88$ & 5.734 & 25,3 \\
Índice circunferência/estatura & & \\
$\quad \leq 0.5$ & 6.958 & 30,8 \\
$\quad>0,5$ & 15.663 & 69,2 \\
Diagnóstico médico de depressão & & \\
$\quad$ Não & 20.752 & 91,7 \\
$\quad$ Sim & 1.869 & 8,3 \\
Saúde autorrereferida & & \\
$\quad$ Muito boa, boa & 962 & 4,3 \\
$\quad$ Ruim, muito ruim ou regular & & \\
\hline
\end{tabular}

em outros estudos que consideram a gravidez e o pós-parto como fatores explicativos de maior prevalência de dores na coluna entre mulheres $^{25,41,42}$. Isso pode ser explicado pelos hormônios da gravidez, como a relaxina, estrógeno e progesterona, que são responsáveis por aumentar a flexibilidade dos ligamentos da coluna e quadril e aumento da lordose lombar, aumento das contraturas musculares devido ao aumento do peso e à postura causados pelo crescimento do feto. No puerpério, o PCC pode ser atribuído às inadequações posturais ao amamentar, ao peso da criança e a outros fatores ${ }^{25,41}$.

Quanto ao IMC, CC e relação C/E verificouse que quanto maiores a massa corporal e adiposidade central, maior a chance de desenvolver PCC, resultado semelhante ao encontrado em outros trabalhos, uma vez que mulheres em idade reprodutiva e com obesidade apresentaram mais queixa de lombalgia quando comparada às eutróficas ${ }^{43}$. De acordo com Malta et al..$^{35}$, o aumento na massa corpórea acarreta sobrecarga na musculatura, processos inflamatórios nos ossos e desgastes do disco vertebral, favorecendo o apa- 
Tabela 2. Fatores associados (OR e respectivos IC95\%) em mulheres que referiram Problema Crônico de coluna, segundo variáveis selecionadas. Pesquisa Nacional de Saúde, Brasil, 2013.

\begin{tabular}{|c|c|c|}
\hline Variável & OR & IC 95\%* \\
\hline \multicolumn{3}{|l|}{ Idade (anos) } \\
\hline $18-26$ & 1 & \\
\hline $27-32$ & 1,39 & $1,22-1,57$ \\
\hline $33-39$ & 1,78 & $1,57-2,00$ \\
\hline $40-49$ & 2,70 & $2,42-3,03$ \\
\hline \multicolumn{3}{|l|}{ Raça/cor } \\
\hline Branca & 1 & \\
\hline Preta, parda e outras & 0,95 & $0,88-1,02$ \\
\hline \multicolumn{3}{|l|}{$\begin{array}{l}\text { Vive com cônjuge ou } \\
\text { companheiro }\end{array}$} \\
\hline Não & 1 & \\
\hline Sim & 1,13 & $1,04-1,22$ \\
\hline \multicolumn{3}{|l|}{ Escolaridade } \\
\hline Sem instrução & 1 & \\
\hline $\begin{array}{l}\text { Até o ensino } \\
\text { fundamental completo }\end{array}$ & 0,84 & $0,74-0,95$ \\
\hline Ensino médio completo & 0,65 & $0,57-0,74$ \\
\hline $\begin{array}{l}\text { Ensino superior } \\
\text { completo ou mais }\end{array}$ & 0,69 & $0,60-0,80$ \\
\hline \multicolumn{3}{|l|}{ Trabalho } \\
\hline Não & 1 & \\
\hline Sim & 1,08 & $1,00-1,16$ \\
\hline \multicolumn{3}{|l|}{$\begin{array}{l}\text { Atividade física nos } \\
\text { últimos } 3 \text { meses }\end{array}$} \\
\hline Não & 1 & \\
\hline Sim & 1,03 & $0,94-1,12$ \\
\hline \multicolumn{3}{|l|}{$\begin{array}{l}\text { Prática de } 150 \text { minutos } \\
\text { de atividade física por } \\
\text { semana }\end{array}$} \\
\hline Não & 1 & \\
\hline Sim & 0,93 & $0,84-1,02$ \\
\hline \multicolumn{3}{|l|}{ Tabagismo } \\
\hline Não & 1 & \\
\hline Sim & 1,63 & $1,46-1,81$ \\
\hline \multicolumn{3}{|l|}{ Hábito de assistir TV } \\
\hline$<2$ horas & 1 & \\
\hline$\geq 2$ horas & 0,93 & $0,86-1,00$ \\
\hline \multicolumn{3}{|l|}{ Número de partos } \\
\hline 0 & 1 & \\
\hline Até 2 & 1,34 & $1,04-1,72$ \\
\hline 3 ou mais & 1,37 & $1,07-1,75$ \\
\hline \multicolumn{3}{|l|}{ IMC } \\
\hline$\leq 24,99$ & 1 & \\
\hline$>24,99 \leq 29,99$ & 1,33 & $1,22-1,45$ \\
\hline$>29,99 \leq 34,99$ & 1,53 & $1,37-1,70$ \\
\hline$>34,99$ & 1,21 & $1,07-1,38$ \\
\hline
\end{tabular}

Tabela 2. Fatores associados (OR e respectivos IC95\%) em mulheres que referiram Problema Crônico de coluna, segundo variáveis selecionadas. Pesquisa Nacional de Saúde, Brasil, 2013.

\begin{tabular}{|c|c|c|}
\hline Variável & OR & IC 95\%* \\
\hline \multicolumn{3}{|l|}{ Circunferência da cintura } \\
\hline$<80$ & 1 & \\
\hline $80-88$ & 1,24 & $1,12-1,37$ \\
\hline$>80$ & 1,46 & $1,33-1,59$ \\
\hline \multicolumn{3}{|l|}{$\begin{array}{l}\text { Índice circunferência/ } \\
\text { altura }\end{array}$} \\
\hline$\leq 0.5$ & 1 & \\
\hline$>0,5$ & 1,51 & $1,39-1,65$ \\
\hline \multicolumn{3}{|l|}{ Autopercepção de saúde } \\
\hline Muito boa, boa & 1 & \\
\hline $\begin{array}{l}\text { Ruim, muito ruim ou } \\
\text { regular }\end{array}$ & 3,58 & $3,13-4,11$ \\
\hline \multicolumn{3}{|l|}{$\begin{array}{l}\text { Diagnóstico médico de } \\
\text { depressão }\end{array}$} \\
\hline Não & 1 & \\
\hline Sim & 3,07 & $2,76-3,41$ \\
\hline
\end{tabular}

recimento de lombalgia e hérnia de disco, entre outras doenças na coluna que são associadas ao PCC $^{35}$.

Sobre autopercepção de saúde, observou-se que as mulheres que referiram autopercepção de saúde ruim, muito ruim ou regular têm 3 vezes mais chances $(\mathrm{OR}=3,58$, IC95\% $=3,13-4,11)$ de desenvolver PCC quando comparadas às mulheres que referiram sua saúde como boa e muito boa. É válido ressaltar que há escassez de estudos sobre o assunto com mulheres em idade reprodutiva, mas resultado semelhante ao encontrado neste trabalho foi apontado na literatura ${ }^{44}$, com mulheres na fase climatérica, quando verificouse que $54 \%$ das mulheres climatéricas avaliadas no estudo que tinham PCC refeririam autopercepção de saúde negativa quando comparadas àquelas que refeririam autopercepção de saúde positiva $^{44}$.

O diagnóstico de depressão foi associado a 3 vezes mais chances de ter PCC, o que pode ser explicado pelas limitações que esta disfunção pode causar. Um estudo realizado com pacientes que sofriam de problema na coluna revelou que $12 \%$ apresentavam depressão em virtude da morbidade, e que a maioria deles tinha expectativa de melhorar com o tratamento ${ }^{45}$.

A escolaridade foi considerada como um fator protetor, em que as mulheres que estudaram até o ensino fundamental completo tinham $84 \%$ 
de fator de proteção ao PCC, seguido das que fizeram o Ensino médio completo (65\%) e Ensino superior completo ou mais (69\%). Dados da Pesquisa Nacional por Amostra de Domicílios ${ }^{34}$ e em um estudo realizado no Sul do Brasil ${ }^{46}$ verificaram que indivíduos menos escolarizados tinham mais dor crônica. Um explicação para isso, segundo Plouvier et al. ${ }^{47}$, é que as pessoas com baixa escolaridade estão mais expostos a más condições de trabalho e por isso desenvolvem mais queixas de PCC quando comparadas às pessoas com maior escolaridade. Segundo dados do Sistema de Vigilância de Doenças Crônicas por Inquérito Telefônico (Vigitel) ${ }^{48}$, inquérito nacional realizado em todas as capitais brasileiras e no Distrito Federal, houve expressiva redução na frequência de autoavaliação negativa da saúde com o aumento da escolaridade ${ }^{48}$.

O presente estudo apresenta algumas limitações por se tratar de um estudo transversal, uma vez que não permite que se façam inferências de causa-efeito em relação ao PCC e às variáveis estudadas. Além disso, ainda há uma relativa escassez de estudos sobre essa temática em mulheres, tanto na idade fértil quanto no climatério.
É válido ressaltar que o termo genérico de Problema Crônico de Coluna utilizado na PNS dificultou os achados para discutir os resultados encontrados neste estudo, uma vez que na literatura estrangeira a maioria dos estudos se referem à dor lombar crônica. Percepção semelhante foi observada em Romero et al. . .

Por meio do estudo pode-se concluir que o PCC acomete 14,8\% das mulheres de idade fértil e está associada à autopercepção negativa de saúde, bem como o avanço da idade, relacionamento com cônjuge/companheiro, tabagismo, multiparidade, sobrepeso e obesidade e diagnóstico de depressão. Já a escolaridade foi o único fator de proteção para o PCC em mulheres na idade fértil.

Portanto, dado que os fatores associados ao PCC podem ser controlados, os resultados apresentados neste estudo podem contribuir para a prevenção do desenvolvimento do PCC reduzindo, assim, os custos com o tratamento dos pacientes no âmbito do sistema único de saúde, bem como para a previdência social, reduzindo o número de afastamentos do trabalho e aposentadoria por invalidez.

\section{Colaboradores}

CVA Oliviera, DE Souza, JPC Silva e GN Correia participaram de todas as etapas de construção e condução da pesquisa e escrita do artigo, AG Magalhães auxiliou na discussão e revisão final do artigo.

\section{Agradecimentos}

À Pró-reitoria de Pós-Graduação da Universidade Federal do Rio Grande do Norte pelo financiado de parte deste estudo. 


\section{Referências}

1. Andersson GB. Epidemiological features of chronic low-back pain. Lancet 1999; 354(9178):581-585.

2. Dionne CE, Dunn KM, Croft PR. Does back pain prevalence really decrease with increasing age? A systematic review. Age Ageing 2006; 35(3):229-234.

3. Hoy D, Brooks P, Blyth F, Buchbinder R. The epidemiology of low back pain. Best Pract Res Clin Rheumatol 2010; 24(6):769-781.

4. Dagenais S, Caro J, Haldeman S. A systematic review of low back pain cost of illness studies in the United States and internationally. Spine J 2008; 8(1):8-20.

5. Deyo RA, Cherkin D, Conrad D, Volinn E. Cost, controversy, crisis: low back pain and the health of the public. Annu Rev Public Health 1991; 12:141-156.

6. Hagen KB, Tambs K, Bjerkedal T. A prospective cohort study of risk factors for disability retirement because of back pain in the general working population. Spine 2002; 27(16):1790-1786.

7. Instituto Brasileiro de Geografia e Estatística (IBGE). Pesquisa Nacional de Saúde. Rio de Janeiro: IBGE; 2014.

8. Meucci RD, Fassa AG, Faria NMX. Prevalence of chronic low back pain: systematic review. Rev Saude Publica 2015; 49.

9. Romero DE, Santana D, Borges P, Marques A, Castanheira D, Rodrigues JM, Sabbadini L. Prevalência, fatores associados e limitações relacionados ao problema crônico de coluna entre adultos e idosos no Brasil. Cad Saude Publica 2018; 34(2):e00012817.

10. Reis RJ, Pinheiro TMM, Navarro A, Martin MM. Perfil da demanda atendida em ambulatório de doenças profissionais e a presença de lesões por esforços repetitivos. Rev Saude Publica 2000; 34(3):292-298.

11. Manek NJ, MacGregor AJ. Epidemiology of back disorders: prevalence, risk factors, and prognosis. Curr Opin Intern Med 2005; 17(2):324-330.

12. Meziat Filho N, Silva GA. Invalidez por dor nas costas entre segurados da Previdência Social do Brasil. Rev Saude Publica 2011; 45(3):494-502.

13. National Institute for Occupational Safety and Health (NIOSH). Musculoskeletal disorders and workplace factors. Cincinnati: NIOSH; 1997.

14. Deyo RA, Weinstein JN. Low back pain. N Engl J Med 2001; 344(5):363-370.

15. Andersson GBJ. Epidemiology of low back pain. Acta Orthop Scand 1998; 281(Supl.):28-31.

16. Kwon M, Shim WS, Kim MH, Gwak MS, Hahm TS, Kim GS, Kim CS, Choi YH, Park JH, Cho HS, Kim TH. A correlation between low back pain and associated factors: a study involving 772 patients who had undergone general physical examination. J Korean Med Sci 2006; 21(6):1086-1091.

17. Wong EY, Deyo RA. Acute low back pain. Primary Care Update for OB/GYNS 2001; 8:171-174.
18. Garcia JBS, Hernandez-Castro JJ, Nunez RG, Pazos MA, Aguirre JO, Jreige A, Delgado W, Serpentegui M, Berenguel M, Cantemir C. Prevalence of low back pain in Latin America: a systematic literature review. Pain Physician 2014; 17(5):379-391.

19. Hoy D, March L, Brooks P, Blyth F, Woolf A, Bain C, Williams G, Smith E, Vos T, Barendregt J, Murray C, Burstein R, Buchbinder R. The global burden of low back pain: estimates from the Global Burden of Disease 2010 study. Ann Rheum Dis 2014; 73(6):968-974.

20. Speroff $\mathrm{L}$. Clinical guidelines for contraception at different ages: early and late. In: Speroff L, Darney PD, editors. A clinical guide for contraception. Philadelphia: Lippincott Williams \& Wilkins; 2011. p. 351-379.

21. Moreno N, Moncada S, Llorens C, Carrasquer P. Double presence, paid work, and domestic-family work. New Solutions 2010; 20(4):511-526.

22. Brasil. Ministério da Saúde (MS). Política Nacional de Atenção Integral à Saúde da Mulher: princípios e Diretrizes. Brasília: MS; 2011. (Série C. Projetos, Programas e Relatórios).

23. Frasson VB. Uso Racional de Medicamentos: fundamentação em condutas terapêuticas e nos macroprocessos da Assistência Farmacêutica. Dor lombar: como tratar? Brasília: OPAS/OMS; 2016.

24. Hansson TH, Hansson EK. The effects of common medical interventions on pain, back function, and work resumption in patients with chronic low back pain: A prospective 2-year cohort study in six countries. Spine (Phila Pa 1976) 2000; 25(23):3055-3064.

25. Ferreira GD, Silva MC, Rombaldi AJ, Wrege ED, Siqueira FV, Hallal PC. Prevalência de dor nas costas e fatores associados em adultos do Sul do Brasil: estudo de base populacional. Rev Bras Fisioter 2011; 15(1):3136.

26. Mata MS, Costa FA, Souza TO, Mata ANS, Pontes JF. Dor e funcionalidade na atenção básica à saúde. Cien Saude Colet 2011; 16(1):221-230.

27. Souza-Júnior PRB, Freitas MPS, Antonaci GDA, Szwarcwald CL. Desenho da amostra da Pesquisa Nacional de Saúde 2013. Epidemiol Serv Saúde 2015; 24(2):207-216

28. Szwarcwald CL, Malta DC, Pereira CA, Vieira MLFP, Conde WL, Souza Junior PRB, Damacena GN, Azevedo LO, Azevedo e Silva G, Theme Filha MM, Lopes CS, Romero DE, Almeida VS, Monteiro CA. Pesquisa Nacional de Saúde no Brasil: concepção e metodologia de aplicação. Cien Saude Colet 2014; 19(2):333342.

29. Brasil. Ministério da Saúde (MS). Portaria no 1.119 de 05 de junho de 2008. Regulamenta a Vigilância de Óbitos Maternos. Diário Oficial da União 2008; 5 jun.

30. World Health Organization (WHO). Global Strategy on Diet, Physical Activity and Health. [acessado 2018 Maio 27]. Disponível em: http://www.who.int/ dietphysicalactivity/publications/recommendations18_64yearsold/en/ 
31. World Health Organization (WHO). Obesity: Preventing and managing the global epidemic. - Report of a WHO consultation on obesity. Geneva: WHO; 2000. [WHO Technical Report Series no 894].

32. Schisterman EF, Faraggi D, Reiser B, Trevisan M. Statistical inference for the area under the receiver operating characteristic curve in the presence of random measurement error. Am J Epidemiol 2001; 154(2):174179.

33. Barros MBA, Cesar CLG, Carandina LT, Graciella D. Desigualdades sociais na prevalência de doenças crônicas no Brasil, PNAD-2003. Cien Saude Colet 2006; 11(4):911-926.

34. Barros MBA, Francisco PMSB, Zanchetta LM, Cesar CLG. Tendências das desigualdades sociais e demográficas na prevalência de doenças crônicas no Brasil, PNAD: 2003-2008. Cien Saude Colet 2011; 16(9):3755-3768.

35. Malta DC, Oliveira MM, Andrade SSCA, Caiaffa WT, Souza MFM, Bernal RTI. Fatores associados à dor crônica na coluna em adultos no Brasil. Rev Saude Publica 2017; 51(Supl. 1):9s.

36. Dutra ML, Prates PL, Nakamura E, Villela WV. A configuração da rede social de mulheres em situação de violência doméstica. Cien Saude Colet 2013; 18(5):1293-1304.

37. Pavão ALB, Werneck GL, Campos MR. Autoavaliação do estado de saúde e a associação com fatores sociodemográficos, hábitos de vida e morbidade na população: um inquérito nacional. Cad Saude Publica 2013; 29(4):723-734.

38. Sá K, Baptista AF, Matos MA, Lessa I. Prevalência de dor crônica e fatores associados na população de Salvador, Bahia. Rev Saude Publica 2009; 43(4):622-630.

39. Webb R, Brammah T, Lunt M, Urwin M, Allison T, Symmons D. Prevalence and predictors of intense, chronic, and disabling neck and back pain in the UK general population. Spine 2003; 28(11):1195-1202.

40. Wijhoven HA, Vet HC, Picavet HS. Explaining sex differences in chronic musculoskeletal pain in general population. Pain 2006; 124(1-2):158-166.

41. Machado GPM, Barreto SM, Passos VMA, Lima-Costa MFF. Projeto Bambuí: prevalência de sintomas articulares crônicos em idosos. Rev Assoc Med Bras 2004; 50(4):367-372.

42. Firmento BS, Moccellin AS, Albino MAS, Driusso P. Avaliação da lordose lombar e sua relação com a dor lombopélvica em gestantes. Fisioter Pesq 2012; 19(2):128-134

43. Goel S, Mani P, Mangla D, Goel JK. Low back ache in working women of reproductive age group. Journal of South Asian Federation of Obstetrics and ginaecology 2015; 7(1):33-36.

44. Silva VH, Rocha JSB, Caldeira AP. Fatores associados à autopercepção negativa de saúde em mulheres climatéricas. Cien Saude Colet 2018; 23(5):1611-1620.
45. Pacola LM, Nepomuceno E, Dantas RAS, Costa HRT Cunha DCPT, Herrero CFPS, Defino HLA. Health-related quality of life and expectations of patients before surgical treatment of lumbar stenosis. Coluna/Columna 2014; 13(1):35-38.

46. Silva MC, Fassa AG, Valle NCJ. Dor lombar crônica em uma população adulta no Sul do Brasil: prevalência de fatores associados. Cad Saude Publica 2004; 20(2):377-385.

47. Plouvier S, Leclerc A, Chastang JF, Bonenfant S, Goldberg MS. Socioeconomic position and low-back pain - the role of biomechanical strains and psychosocial work factors in the GAZEL cohort. Scand J Work Environ Health 2009; 35(6):429-436.

48. Brasil. Ministério da Saúde (MS). Vigitel Brasil 2014: vigilância de fatores de risco e proteção para doenças crônicas por inquérito telefônico. Brasília: MS; 2015.
Artigo apresentado em 05/06/2018 Aprovado em 25/07/2018

Versão final apresentada em 27/07/2018 
